\title{
Revisiting aqueous alkaline silicates as precursors for sol-gel optical coatings
}

Anaël Jaffrès, ${ }^{1}$ Mickael Boudot, ${ }^{1}$ Sylvain Chevalier, ${ }^{1}$ Rabei Mohammedi, ${ }^{1}$ Sebastien Maron, ${ }^{1}$ Lucie Devys, ${ }^{2}$ and Thierry Gacoin ${ }^{1}$

${ }^{1}$ Laboratoire de Physique de la Matière Condensée, UMR 7643, Ecole Polytechnique, CNRS, Université Paris-Saclay, Route de Saclay, 91128 Palaiseau cedex (France)

${ }^{2}$ Département Couches Minces, Saint-Gobain Recherche, 39 quai Lucien Lefranc, 93303 Aubervilliers, (France)

Corresponding Author: thierry.gacoin@polytechnique.edu

\begin{abstract}
Alkaline silicates are well known soluble silica derivatives already widely used for industrial purposes. Nevertheless, their applications for the elaboration of functional optical coatings have largely been discarded by the sol-gel community who mainly focused on alkoxide precursors. The objective of this work is to reconsider the alkaline silicates sol-gel chemistry, targeting optical coatings of a few hundreds of nanometers. The structures of $\mathrm{Li}^{+}, \mathrm{Na}^{+}$and $\mathrm{K}^{+}$silicate coatings are discussed on the basis of spectroscopic characterizations. We show that a simple washing process allows a post-deposition removal of most alkaline ions, thus preventing the efflorescence effect and improving the aqueous stability of the films. Finally, several examples are shown for the application of alkaline silicates in the elaboration of functional optical coatings following conventional sol-gel chemistry.
\end{abstract}




\section{Introduction}

Sol-gel chemistry has been the subject of numerous investigations motivated by new low-temperature reaction pathways toward inorganic or hybrid organic/inorganic materials [1]. This allows for the development of compounds with original structures at various scale associated with low-cost shaping processes such as liquid deposition of functional coatings. Still being an intense field of research, developments up to the industrial scale have been achieved for various applications such as paintings, antireflective coatings, photocatalysis...[2-4] Among all possible compositions related to functional oxides, silica can by far be considered as the emblematic compound of the sol-gel chemistry [5]. This is explained by specifically appropriate physical-chemical properties of this compound allowing an almost perfect control of the sol-gel hydrolysis/condensation reactions, high colloidal stability, and opportunities given by the stable Si-C bound for the development of hybrid organic/inorganic compositions. Regarding applications, sol-gel silica (either dense or porous) is usually considered as a platform for surface immobilization of active species, or as a binder or a host matrix for functional species (pigments, nanoparticles, molecules...) [6-7]. The standard precursor for silica is a silicon alkoxide (tetraethylorthosilicate, TEOS) in acid or basic water/alcohol mixtures. Interestingly, there are relatively few academic reports on soluble alkaline silicates as a potential silica precursor for sol-gel coatings alternate to TEOS, although these precursors are low-cost, industrially available and stable over time [811]. These compounds are nevertheless already intensively used for industrial applications such as cement additive, anti-corrosion coatings, flame-retardant coatings, adhesives and paint binders...[12-14] The present work aims at revisiting the materials chemistry of alkaline silicates for applications in the elaboration of optical coatings (typically a few hundreds of $\mathrm{nm}$ thick), as usually achieved using standard alkoxysilane TEOS formulations. Starting from commercial products (lithium, sodium and potassium silicates), we investigate the possibility to prepare coatings by a liquid deposition process with a good optical homogeneity, a controlled structure and a good time durability. In particular, the key-parameters controlling the coatings structure (at macro/microscopic scales) are discussed as well as the evaluation and improvement of their durability. Finally, their potential use as binders for functional additives such as nanoparticles or porogen agents, or for the elaboration of nanostructured coatings is presented. 


\section{Experimental Procedure}

Materials. The studied systems are commercially available basic silicates sols noted by the silicon concentration [Si] and the generic formula $\mathrm{M}_{\mathrm{x}} \mathrm{SiO}_{(2+\times / 2)}\left(\mathrm{M}^{+}: \mathrm{Li}^{+}, \mathrm{Na}^{+}, \mathrm{K}^{+}\right)$, x corresponding to the $\mathrm{M}_{2} \mathrm{O} / \mathrm{SiO}_{2}$ molar ratio) (Table 1).

All these products were used as purchased. The "reference" silica sol was prepared by acid hydrolysis of a commercial tetraethylorthosilicate (TEOS, 98\% from Aldrich) [15]. Unless otherwise specified, experiments were achieved after dilution of the commercial solution with doubly distilled water to set to $1.5 \mathrm{M}$ the silica concentration for all investigated sols.

${ }^{29} \mathrm{Si}$ liquid NMR. The structures of the basic silicates in solution were analyzed by liquid ${ }^{29} \mathrm{Si} \mathrm{NMR}$, giving access to the different $Q^{n}(n=0, \ldots, 4)$ species [16]. Spectra were recorded using a $300 \mathrm{MHz}$ Avance Bruker spectrometer. Heavy water ( $20 \%$ in volume) was added to the samples for locking. A pulse width of $90^{\circ}(11 \mu \mathrm{s})$ with a repetition time optimized at $10 \mathrm{sec}$ was selected. Contribution from silica of the probe and the sample tube were corrected by substraction of the signal from a blank sample recorded under the same experimental conditions.

Preparation of the silicate coatings. The $1.5 \mathrm{M}$ prepared silicates and TEOS sols were prepared and spin-coated at 2000 rpm during 60s on UV-Ozone pre-cleaned silicon wafers. For every formulation, half of the coated samples were dried at ambient temperature after coating and half were annealed at $450^{\circ} \mathrm{C}$ for $2 \mathrm{~h}$ (heating rate of $5^{\circ} \mathrm{C} / \mathrm{min}$ ).

Washing step of the films for alkaline ions removal. Partial removal of the alkaline ions is achieved by a simple washing of the films after deposition and drying, and before any thermal treatment. This is done simply by flowing distilled water on the surface of the film for about 20 seconds. The film is then dried under nitrogen flow.

Characterizations of the coatings: FT-IR, SEM, XRD, ellipsometry. The optical quality of the coatings was first characterized using a Nikon optical microscope with a x40 magnification.

Scanning Electron Microscopy (SEM) characterizations were achieved on samples coated with a few $\mathrm{nm}$ of $\mathrm{Pt}$ (Hitachi E-1030 ion-coater) to avoid charge accumulation. Cross sections and plane views were 
observed on a Hitachi S4800 scanning electron microscope (field emission source, $5 \mathrm{kV}$ acceleration voltage).

The coating thicknesses were confirmed by ellipsometry measurements performed using a MM-16 Horiba Jobin Yvon ellipsometer operating in the visible range $(450-1000 \mathrm{~nm})$. This technique also provides the refractive index and the porous fraction of the silicate layer using a Sellmeier transparent function to simulate the experimental data [17].

X-Ray diffraction patterns for the determination of crystalline phases (surface precipitates) were acquired using a Panalytical (Philips) X'Pert diffractometer operating with Cu Ka radiation (1.5418 A, Ka1+Ka2).

Chemical composition and structural properties of the coatings were analyzed by FTIR spectroscopy operating in transmission mode. Spectra were collected on a Bruker Equinox 55 spectrometer subtracting the silicon wafer absorption as the background.

${ }^{29} \mathrm{Si}$ solid-state MAS NMR. The structure of the dried silicates powders was analyzed by solid NMR ${ }^{29} \mathrm{Si}$, giving access to the different $Q^{n}(n=0-4)$ species [18]. The solid-state ${ }^{29}$ Si magic-angle spinning (MAS) NMR spectra were obtained using an Apollo (Tecmag) spectrometer (magnet WB 8.48 T) using $7 \mathrm{~mm}$ Teflon rotors with $4 \mathrm{kHz}$ spinning speed (silicon resonance frequency at $71 \mathrm{MHz}$ ). A pulse width of 3.55 $\mu$ s with a repetition time optimized at $20 \mathrm{sec}$ was identified. The alkaline silicates were mixed to a solution of chromium acetate in methanol (typically $100 \mu \mathrm{l}$ in $3 \mathrm{ml}$ ) as a relaxant compound. The solution was then dried in an oven $\left(90-100^{\circ} \mathrm{C}\right.$ during 1 night). The obtained solid was crushed in a mortar before analysis. NMR experiments performed on dried silicate with and without chromium acetate led to identical spectra and then confirmed that adding this relaxant compound had no influence on the silicate structure. In addition, the validity of this technique as a probe for the coating structure was confirmed by doing NMR spectra of lithium silicate powders from grinded dried bulk silicate and from a multi-layer coating (Suppl. Info, figure SI-1).

\section{Results and Discussion}


The commercial alkaline silicate solutions (diluted to have a [Si] concentration of $1.5 \mathrm{M}$ ) were spin-coated on silicon substrates in order to obtain homogeneous coatings. All samples exhibited a thickness of about 150 to $180 \mathrm{~nm}$ (Figure 1), this thickness being mainly determined by the precursor concentration.

\section{(a) Influence of the structure of the starting precursor solutions on the coatings structure}

First part of our work was devoted to the investigation of the structure of the coatings, especially focusing on the silica network as probed by IR and NMR spectroscopy. A first issue was to achieve liquid ${ }^{29} \mathrm{Si}$ NMR characterizations of the precursor solutions as a function of the nature of the alkaline ions $(M=N a, L i, K)$ and their relative concentration (expressed in term of $\mathrm{x}=\mathrm{M}_{2} \mathrm{O} / \mathrm{SiO}_{2}$ molar ratio). The idea was to determine the structure of the alkaline silicates clusters of basic silicates solutions and find out some possible correlations with the structure of the silica network in the final dried coating. Results are presented on Figure 2.

Main peaks observed in all spectra correspond to $Q^{0}$ ( $-72 \mathrm{ppm}$, monomers), $Q^{1}$ (-81 ppm, end-groups of chains), $\mathrm{Q}^{2 \Delta}$ ( - $83 \mathrm{ppm}$, cycles), $\mathrm{Q}^{2,3 \Delta}$ (- $89 \mathrm{ppm}$, middle groups of chains or cycles), $\mathrm{Q}^{3}$ (- $98 \mathrm{ppm}$, chain branching sites) and $Q^{4}$ (colloidal species, -108 ppm) 19-20].

The ${ }^{29} \mathrm{Si}$ NMR spectra of $\mathrm{Na}$ and $\mathrm{K}$ silicates with the same amount of alkaline ions $(\mathrm{x}=0.33)$ are plotted in Figure 2a.

The same peaks are observed on the two spectra with similar intensities at the exception of the $Q^{0}$ peak. The latter is slightly more intense for $\mathrm{K}^{+}$, meaning that this solution could contain more monomers. Nevertheless, as a first approximation, not much influence is found regarding the nature of the $\mathrm{M}$ cation $\left(\mathrm{Na}^{+}\right.$or $\left.\mathrm{K}^{+}\right)$on the solution structure. Since the $\mathrm{Li}^{+}$solution was not commercially available with the same ratio $x(0.33)$, the influence of lithium cations on the solutions structure was studied by mixing potassium $(x=0.26)$ and lithium $(x=0.4)$ solutions (with a final ratio $x$ fixed at 0.33$)$. The obtained NMR spectrum differs in the peaks relative intensities, mostly between the peaks $Q^{2,3 \Delta}$ and $Q^{3}$ (Figure 2a). In addition, a weak peak appears between 100 and 110 ppm, attributed to colloidal $\mathrm{Q}^{4}$ species. Therefore, the addition of lithium ions influences the solution condensation towards more condensed $Q^{3}$ and $Q^{4}$ species, in agreement with the results obtained in [21]. This specific behavior was assigned to the specific interactions between lithium ions and silicates, leading to more condensed cluster species. We note that 
this is coherent with the availability of stable lithium silicates sols with lower $x$ ratios than other alkaline ions that tend to become very viscous or even turn into gels while decreasing $\mathrm{x}$.

The silicate solutions are then spin-coated and their structures are analyzed by FTIR (Figure 2b) allowing to discuss the silicate network structure. FTIR has been shown in many previous studies (eg [22-23]) to provide qualitative information on the condensation state of the silica network, especially considering the SiOSi band at $1000-1200 \mathrm{~cm}^{-1}$ and the SiO- band around $950 \mathrm{~cm}^{-1}$. In particular, a frequency increase of the band peaking at $1000-1075 \mathrm{~cm}^{-1}$ (corresponding to the asymmetric stretching mode of SiOSi groups (TO)) suggests a strengthening of the silicate network by condensation of the silanol groups.

The sodium and potassium coatings present their main SiOSi bands with a similar shape centered around $1000 \mathrm{~cm}^{-1}$, evidencing a similar condensation state. In contrast, this band is clearly shifted to higher wavenumbers $\left(1025 \mathrm{~cm}^{-1}\right)$, in the case of potassium/lithium coating, reflecting a denser silica network [22]. This is in accordance with the sol structure that thus seems to be preserved after deposition, i.e. after solvent evaporation, sol concentration and associated silanol condensation. Comparison of all previous compositions with the TEOS reference show that the latter, having its $\mathrm{Si}-\mathrm{O}-\mathrm{Si}$ band at much higher wavenumber $\left(\sim 1075 \mathrm{~cm}^{-1}\right)$, is much more condensed still in accordance with the initial sol structure. This also results from condensation reactions occurring during solvent removal, which are intrinsically limited in the presence of the alkaline ions that act as network modifiers similarly as in glass [22].

Now considering the influence of the $x$ value, two lithium silicate sols with different $x$ ratio $(x=0.2$ and 0.4$)$ were characterized. The corresponding ${ }^{29}$ Si NMR spectra are plotted in Figure 2c. The main difference is the relative intensity of the $Q^{2,3 \Delta}$ and $Q^{3}$ peaks. In addition, when $x$ decreases from 0.4 to 0.2 , a broad peak appears between 100 and 110 ppm, attributed to colloidal $Q^{4}$ species while the $Q^{2 \Delta}$ peak vanishes. Therefore the decrease of $\mathrm{Li}_{2} \mathrm{O}$ content logically influences the solution condensation towards $\mathrm{Q}^{3}$ and $\mathrm{Q}^{4}$ species, in agreement with the results reported in [21]. These silicate coatings are then analyzed by FTIR (Figure $2 \mathrm{~d}$ ). While decreasing the $\mathrm{x}$ ratio (i.e. decreasing the amount of lithium ions), the SiOSi band shifts to higher wavenumbers (from 1020 to $1040 \mathrm{~cm}^{-1}$ ), reflecting a denser silica network. This tendency observed in the solution structure is then preserved after coating and the basic silicate coatings still 
appear less condensed than the reference TEOS derived coating.

${ }^{29} \mathrm{Si}$ solid NMR spectra of the dried silicate powders were also measured to get complementary information on the films structures through the repartition of the different $Q^{n}$ species. Results, given in Table 2, confirm the conclusions of the IR spectroscopy analysis (data of NMR spectra are gathered in Suppl. Info figures SI-2,3).

\section{(b) Influence of a thermal treatment on the coatings structure}

The influence of a thermal treatment on the coatings structure is studied by annealing the ambient silicate coatings at $450^{\circ} \mathrm{C}$ for 2 hours. The corresponding IR spectra are displayed in Figure $2 \mathrm{~b}, \mathrm{~d}$ (dashed lines) and the repartition of $Q^{n}$ silicate species of dried and dried/annealed silicate powders are gathered in Table 2. Figure 3 presents the shift of the maximum wavenumber of the SiOSi band while annealing the silicate coatings.

For all silicates, an annealing step contributes to the silica densification as highlighted by the shift of the

SiOSi band (around 1000-1075 $\mathrm{cm}^{-1}$ ) towards higher wavenumbers and/or by the decreased intensity of the $950 \mathrm{~cm}^{-1} \mathrm{SiO}^{-}$band observed in Figure $2 \mathrm{~b}$ and $\mathrm{d}$.

This is confirmed by solid-state NMR spectra of the dried/annealed solutions of basic silicate and TEOS (Table 2 and Suppl. Info figures SI-2,3). For TEOS, the dried powder contains mostly $\mathrm{Q}^{3}$ species whereas the annealed powder presents only $\mathrm{Q}^{4}$ species (i.e a fully condensed silica network). We observe a similar phenomenon on annealed lithium silicate powder $(x=0.2)$ : the initial mix of $Q^{3}$ and $Q^{4}$ species of the dried powder turns into a majority of $Q^{4}$ species. We also note the appearance of a peak attributed to $Q^{0}$ species showing the thermally induced segregation of the $\mathrm{Li}$ ions, a phenomenon already observed in lithium silicate glasses in the same range of composition [23]. In the case of other alkaline ions ( $\mathrm{Na}, \mathrm{K})$, the densification also occurs but to a lower extend probably due to a lower mobility of these ions that remain distributed among the silica network.

\section{(c) Ageing of the basic silicate coatings}


The surfaces of the silicate coatings with different alkaline cations $(\mathrm{Na}, \mathrm{Li}, \mathrm{K}$, mixing of $\mathrm{K}$ and $\mathrm{Li})$ are displayed in Figure 4 two weeks and three months after spin-coating. The TEOS reference coating is also shown for comparison. A quick evolution of their aspect (Figure 4a-d) is observed with a strong influence of the nature of the alkaline cation. After three months, all alkaline doped silicate coatings present micronsize defects on their surface (Figure 4f-i).

These observations correspond to the phenomenon of efflorescence (or carbonation) that corresponds to the formation of alkaline carbonates at the surface of the films. These carbonates are formed through the reaction of alkaline ions migrating at the surface of the film with atmospheric $\mathrm{CO}_{2}$ [24]. It must be noted that a post-deposition annealing step does not prevent this effect but just slows down its kinetics over several months due to the slower diffusion of the ions within the more condensed silicate matrix (figure 4). In the case of the TEOS reference coating, no efflorescence effect is observed and the film remains homogeneous over time (Figure 4e,j).

A FTIR kinetic study was carried out over several months in order to investigate the durability of the silicate coatings. The characterizations done on aged sodium silicate coatings are presented in Figure 5 as well as a comparison with TEOS-based coatings (additional characterizations on aging are gathered in Suppl. Info figures SI-4,5).

SEM observations of a one month-old sodium silicate coating cross section reveals the presence of needle-shaped crystals on the top of the coating (Figure 5b). Those crystals, characterized by grazing incidence XRD (incidence angle $3^{\circ}$ ) exhibit the sodium carbonate phase (JCPDS file 9-011-304) as expected (Figure 5a).

The FTIR spectra of a two-month sodium silicate coating at different times of ageing prove that carbonation occurs almost immediately after coating as proved by the IR bands at $880 \mathrm{~cm}^{-1}$ and $1425 \mathrm{~cm}^{-}$

${ }^{1}$ (Figure 5c). The sodium carbonate crystals evolve after a few weeks into sodium hydrogenocarbonate (Figure $5 \mathrm{c}$, light blue curve). In the meantime, the band attributed to SiOSi (around $1000 \mathrm{~cm}^{-1}$ ) shifts towards higher wavenumbers (Figure 3), showing a significant silica network condensation over time.

We observe similar phenomenon (carbonation and silica condensation) for potassium and lithium coatings although their kinetics are very different. The potassium silicate coatings display carbonation 
immediately after coating with the direct formation of potassium hydrogenocarbonate crystals (detected by IR and XRD). Being hygroscopic, these crystals solubilize partially under ambient humidity as showed by FTIR (see Suppl. Info figure SI-4). The lithium silicate coatings present a much slower but effective carbonation kinetic as the lithium carbonate crystals are only spotted after two months of study (Suppl. Info figure SI-5). This carbonation is also associated with a condensation of their silica network (shift of the SiOSi IR band towards higher wavenumbers, Figure 3), but not as strong as observed for sodium (Figure $5 \mathrm{c}$ ). Although $\mathrm{Na}$ and $\mathrm{K}$ coatings present a similar average condensation just after spin-coating, the latter coatings displays a lower densification after two months.

Annealing the coatings at $450^{\circ} \mathrm{C}$ for 2 hours does not influence much the phenomenon of carbonation and condensation observed on the ambient coatings over time (see Suppl. Info figure SI-6).

Moreover, the TEOS-based reference coatings do not change in the time after the coating, in terms of aspect, carbonation, and silica network condensation (Figure 5d).

\section{(d) Improvement of durability of basic silicate coatings by a simple water-washing process}

Despite the interest of the precursor, the efflorescence phenomena and the solubility of alkaline silicates are severe drawbacks for their practical use for functional coatings. In order to limit those phenomena, it seems interesting to investigate the possibility of a post-deposition removal of the alkaline ions. Acid washing of aged silicates films obviously removes the surface carbonates, but we interestingly found that a simple washing with water just after deposition leads to similar results, considering both the carbonation effect and the resulting condensation of the silica network. Note that this washing does not dissolve the film, which thickness remains almost unchanged within $5 \%$ as checked by ellipsometry and SEM (see Suppl. Info figure SI-7).

Figure 6a presents the IR spectra of a lithium silicate coating after different successive steps : just spincoated, after washing with water, and after a further annealing at $450^{\circ} \mathrm{C}$. A rapid water-washing of the film immediately after coating leads to the condensation of the silica network (shift of the SiOSi band, Figure 6a) through removal of most of its lithium content as observed by ToF-SIMS experiments (Suppl. Info figure SI-8). The IR spectrum of the resulting coating is very similar to the one of TEOS, our reference 
sample. This result is very interesting since we can take benefit of the alkaline silicate precursors (sol stability, low cost, water formulation...) and obtain, through a very simple process, coatings that are similar to the TEOS-based ones.

The FTIR spectra of water-washed then annealed silicate coatings for different compositions are displayed in Figure 6b. All spectra are similar with the presence of bands characteristic of SiOSi at 825 $\mathrm{cm}^{-1}$ and $1075 \mathrm{~cm}^{-1}$, meaning their local structure is similar regardless of the starting composition of the silicate solution and almost identical to the TEOS coatings.

While the IR spectra presented above provide information on the local structure of silicate coatings the question is raised to determine how the local densification of the silicate coating after washing/annealing impacts the structure of the film at a larger scale, for example regarding the concomitant formation some porosity [25-26]. The films were characterized by ellipsometry for their thickness and refractive index. Results for as-deposited, water-washed and then annealed samples are presented in Figure 6c. The refractive index of the just-deposited coatings $(n=1.465-1.470)$ are higher than the reference value obtained for an annealed TEOS coating $(n=1.44))$ due to the presence of the alkaline ions [27]. After washing, the refractive indexes largely decrease as a result of the alkaline ions removal, leaving a partially hydroxylated silica network. Annealing at $450^{\circ} \mathrm{C}$ allows condensing the network through dehydroxylation, leading to an increase of the refractive index. After this step, the values of refractive indexes spread from 1.425 to 1.45 depending on the nature of the alkaline ion and the $\mathrm{x}$ value. The final values result mainly from a balance between residual porosity and the presence of residual alkaline ions that respectively decrease and increase the refractive index as compared to pure silica. The case of $\mathrm{K}^{+}$ leads to a refractive index higher than pure silica, which shows that a significant amount of $\mathrm{K}^{+}$ions are remaining in the film due to a lower mobility of this ion. In the case of $\mathrm{Li}^{+}$and $\mathrm{Na}^{+}$, refractive index values of 1.435 and 1.425 are measured respectively, lower than pure sol-gel silica so that significant porosity is expected. TOF-SIMS analysis shows that lithium ion concentration in the $\mathrm{Li}^{+}$film is negligible, since at least $10^{3}$ less than initially. Therefore, assuming the complete removal of alkaline earth ions, the lower refractive index observed for $\mathrm{Li}^{+}$film indicates that is composed of a porosity that can be estimated to 
about $10 \%$. In the case of sodium, the refractive index is even lower, corresponding to a porosity of at least $35 \%$, which is an underestimated value due to the possible presence of remaining sodium ions.

Finally, an ageing study is performed on such coatings as well as on reference silicate coatings during 6 months (Figure 6d). The main result of this study is that water-washed coatings present carbonation close to zero even after six months, unlike reference silicate coatings. This confirms the efficiency of the simple washing for stabilization the coating toward carbonation.

\section{(e) Applications of silicate coatings}

Regarding their obvious advantages as compared to the TEOS standard precursor (low cost, in aqueous medium, stable over time...), it is interesting to discuss on the use of these kinds of precursors for some classical applications of sol-gel silica coatings.

A first possibility is the use of silicate as binders for nanoparticulate coatings, ensuring good nanoparticles dispersion and sufficient mechanical properties. As a proof of concept, we considered luminescent $\mathrm{YVO}_{4}$ :Eu nanoparticles that are obtained as aqueous colloidal suspensions through conventional colloid chemistry [29]. Nanoparticulate coatings were prepared through mixing a $\mathrm{YVO}_{4}$ colloidal suspension $(0.1$ mol. $\mathrm{L}^{-1}$ expressed in term of $\mathrm{VO}_{4}{ }^{3-}$ concentration) with a 2.4 mol. $\mathrm{L}^{-1}$ lithium silicate precursor $(\mathrm{x}=0.25)$. The relative volume fraction of each solution was taken as 90:10 respectively. Interestingly, the silicate allows for an improved stabilization of the YVO4:Eu particles which zeta potential increased from $-19 \mathrm{mV}$ to -38 $\mathrm{mV}$, leading to highly dispersed particles in the silicate solution (Figure 7a). After spin coating on a silicon substrate, homogeneous photoluminescent coatings are obtained as presented in Figure $7 \mathrm{~b}$.

Similar experiments of nanocomposite coatings deposition have been performed using Ludox ${ }^{\mathrm{R}}$ silica nanoparticles. Highly homogeneous films could be obtained whatever their volume fraction ranging from 0 to $100 \%$ with respect to the silicate (Suppl. Info figure SI-9).

Mesostructured TEOS precursor-based silica films are another class of sol gel material that has known a great interest since the 90's, with applications in optics, electronics, photocatalysis, sensing, etc...[15,3031]. As a silicate-based alternative, mesoporous films were synthesize from a solution prepared by adding dropwise under stirring $15 \mu \mathrm{L}$ of lithium silicate $(2.4 \mathrm{M})$ into $5 \mathrm{~mL}$ of a PMMA latex solution at 
concentration of $2.95 \mathrm{~g} / \mathrm{L}$. PMMA beads with an average size of $65 \mathrm{~nm}$ in diameter were chosen. Then solution was spin coated at $1000 \mathrm{rpm}$ for $1 \mathrm{~min}$, washed with water and annealed at $450^{\circ} \mathrm{C}$ for $10 \mathrm{~min}$. Latex-templated mesoporous films exhibit after calcination an average thickness and a refractive index of $125 \mathrm{~nm}$, and 1.22 (at $700 \mathrm{~nm}$ ) respectively, which corresponds to a porous volume of $45 \%$. As shown in Figure 8, mesopores, with a mean size of $45 \mathrm{~nm}$, are homogeneously distributed in silica films, evidencing the fairly good PMMA beads dispersion in solution. By modifying the PMMA beads size and concentration, mesostructured silica films with a large range of porous volume and structure can be fabricated [15].

As a third example, we investigated the use of lithium silicate for the preparation of patterned coatings as obtained by embossing with a PDMS stamp following the MIMIC (Micromolding in Capillaries) process [32]. The PDMS stamp was obtained starting from a silicon master patterned through electronic lithography. The PDMS stamp is just placed onto a droplet of Li0.2 silicate precursor previously deposited on a glass slide. Drying is achieved in an oven at $60^{\circ} \mathrm{C}$ for $12 \mathrm{~h}$ leading to the patterned coating shown on Figure 9.

\section{Conclusion}

This work was devoted to the investigation of the potential applications of alkaline silicates for the elaboration of sol-gel functional coatings. These precursors are highly appealing due to their commercial availability as aqueous solutions, excellent stability over aging and low cost as compared for example with the commonly used TEOS (tetraethoxysilane).

A first part was devoted to the investigation of the structure of the films, considered mainly in term of local condensation state of the silica network as a function of the nature of the alkaline ion $(M=N a, L i, K)$ and the relative $\mathrm{M}_{2} \mathrm{O} / \mathrm{SiO}_{2}$ molar ratio $(\mathrm{x})$. It is shown that, at microscopic scale, the structure is controlled both by the microstructure of the spin-coated silicate solution (depending on the nature of the alkaline cation $\mathrm{M}$ and the value of $x$ ) and by a thermal treatment. Hence the densest coatings are obtained from lithium 
silicates and the smallest $\mathrm{x}$ ratio. An annealing step contributes also for densifying the coatings with TEOS $\mathrm{Li}>\mathrm{Na}, \mathrm{K}$ but does not change the structural differences observed on the ambient coatings.

The second part is devoted to ageing of the silicate films leading to the surface precipitation of alkaline carbonates and a significant condensation of the silica network. This so-called efflorescence phenomenon appears to be kinetically limited, but still present when considering the lithium silicate. Interestingly, we show that a simple post-deposition washing with pure water leads to films which condensation state appears to be very similar to the one of common TEOS coatings. Finally, an application of this process is shown in the case of the deposition of silica/particle nanocomposites, considering luminescent $\mathrm{YVO}_{4}: \mathrm{Eu}$ nanoparticles. The alkaline silicate is shown to stabilize the nanoparticles suspension preserving the good dispersion, so that luminescent films with excellent optical quality could be obtained. Further work is under investigation regarding the use of alkaline silicates for the elaboration of mesoporous thin films, or as host matrices for organic dyes, which are other well-known examples of sol-gel silica for optical applications.

\section{Acknowledgements}

TG wishes to thank A. Huignard for his precursor experiments on silicate coatings.

\section{References}

[1] Hench LL, West JK. The Sol-Gel Process. Chem. Rev. 1990; 90: 33-72.

[2] Allain E, Besson S, Durand C, Moreau M, Gacoin T, Boilot JP. Transparent mesoporous nanocomposite films for self-cleaning applications. Adv. Funct. Mater. 2007; 17:549-554.

[3] Pardo R, Zayat M, Levy D. Photochromic organic-inorganic hybrid materials. Chem. Soc. Rev. 2011; 40:672-687.

[4] Shang HM, Wang Y, Limmer SJ, Chou TP, Takahashi K, Cao GZ. Optically transparent superhydrophobic silica-based films. Thin Solid Films 2005; 472:37-43.

[5] Iler RK. The Chemistry ot Silica. New. York, PA: John Wiley and Sons; 1979

[6] Sanchez C, Chaput F, Boilot JP. Optical properties of functional hybrid organic-inorganic nanocomposites. Adv. Mater. 2003; 15 (23):1969-1995.

[7] Penard AL, Gacoin T, Boilot JP. Acc. Chem. Res. 2007; 40(9):895-902

[8] Choi JS, Lee HK, An SJ. Synthesis of a graphene oxide/sodium silicate nanocomposite using sodium silicate solution. RSC Adv. 2015; 5:38742-38747. 
[9] Calderón-Guillén JA, Avilés-Arellano LM, Pérez-Robles JF, González-Hernández J, RamosRamírez E. Dense silica-based coatings prepared from colloidal silica. Surf. Coat. Technol. 2005; 190:110-114.

[10] Sarawade PB, Kim JK, Hilonga A, Quang DV, Kim HT. Effect of drying technique on the physicochemical properties of sodium silicate-based mesoporous precipitated silica. Appl. Surf. Sci. $2011 ;$ 258:955-961.

[11] He J, Fujikawa S, Kunitake T, Nakao A. Preparation of porous and nonporous silica nanofilms from aqueous sodium silicate. Chem. Mater. 2003; 15:3308-3313.

[12] Fraci AT. Waterborne Silicates in Coatings and Construction Chemicals. In: The Australasian Corrosion Association Inc editor. Inorganic Zinc Coatings, History, Chemistry, Properties, Applications and Alternatives. Kerrimuir Victoria, 2013; 227-258.

[13] Pereyra AM, Giudice CA, Flame-retardant impregnants for woods based on alkaline silicates. Fire Saf. J. 2009; 44:497-503.

[14] Vesely D, Kalenda P, Nemec P. Nanoparticles of soluble alkaline silicates as corrosion inhibitors in water based polymer dispersions. Mat.Res. Innovations. 2009; 13:2-4.

[15] Guillemot F, Brunet-Bruneau A, Bourgeat-Lami E, Gacoin T, Barthel E, Boilot JP. Latex-templated silica films: Tailoring porosity to get a stable low-refractive index. Chem. Mater. 2010; 22:28222828.

[16] Halasz I, Kierys A, Goworek J, Liu H, Patterson RE. 29Si NMR and Raman glimpses into the molecular structures of acid and base set silica gels obtained from TEOS and Na-silicate. J. Phys. Chem. C. 2011; 115:24788-24799.

[17] Fujiwara H, Spectroscopic Ellipsometry: Principles and Applications. Chichester, John Wiley \& Sons Ltd; 2007.

[18] Maekawa H, Maekawa T, Kawamura K, Yokokawa T. The Structural Groups of Alkali SilicateGlasses Determined From Si-29 MAS-NMR. J. Non. Cryst. Solids. 1991; 127 53-64.

[19] McCormick AV, Bell AT, Radke CJ. Quantitative determination of siliceous species in sodium silicate solutions by silicon-29 NMR spectroscopy. Zeolites. 1987; 7:183-190.

[20] Harris RK, Bahlmann EKF, Metcalfe K, Smith EG. Quantitative silicon-29 NMR investigations of highly concentrated high-ratio sodium silicate solutions. Magn. Reson. Chem. 1993; 31:743-747.

[21] Gaboriaud F, Nonat A, Chaumont D. ${ }^{29}$ Si NMR and Small-Angle X-ray Scattering Studies of the Effect of Alkaline lons ( $\mathrm{Li}_{+}, \mathrm{Na}+$, and $\mathrm{K}+$ ) in Silico-Alkaline Sols. J. Phys. Chem. B 1999; 103:2091-2099.

[22] Kamitsos El, Patsis AP, Kordas G. Infrared-reflectance spectra of heat-treated sol-gel-derived silica. Phys. Rev. B. 1993; 48:12499-12505.

[23] Vogel W, Structure and Crystallization of Glasses, Leipzig, PA : Pergamon Press; 1971 21-127.

[24] Veinot DE, Langille KB, Nguyen DT, Bernt JO. Efflorescence of soluble silicate coatings. J. Non. Cryst. Solids. 1991; 127:221-226.

[25] Huang QZ, Shi JF, Wang LL, Li YJ, Zhong LW, Xu G. Study on sodium water glass-based antireflective film and its application in dye-sensitized solar cells. Thin Solid Films. 2016; 610:19-25.

[26] Nielsen KH, Orzol DK, Koynov S, Carney S, Hultstein E, Wondraczek L. Large area, low cost antireflective coating for solar glasses. Sol. Energy Mater. Sol. Cells. 2014; 128:283-288.

[27] Jen JS, Kalinowski MR. An ESCA study of the bridging to non-bridging oxygen ratio in sodium silicate glass and the correlations to glass density and refractive index. J. Non. Cryst. Solids. 1989; 38-39:21-26. 
[28] Boudot M, Gaud V, Louarn M, Selmane M, Grosso D. Sol-Gel Based Hydrophobic Antireflective Coatings on Organic Substrates: A Detailed Investigation of Ammonia Vapor Treatment (AVT). Chem. Mater. 2014; 26:1822-1833.

[29] Huignard A, Gacoin T, Boilot JP. Synthesis and luminescence properties of colloidal YVO4 : Eu phosphors. Chem. Mat. 2000; 12:1090-1094.

[30] Boudot M, Elettro H, Grosso D. Converting Water Adsorption and Capillary Condensation in Usable Forces with Simple Porous Inorganic Thin Films. ACS Nano 2016; 10(11):10031-10040.

[31] Hatton BD, Landskron K, Hunks WJ, Bennett MR, Shukaris D, Perovic DD et al. Materials chemistry for low-k materials. Mater. Today. 2006; 9:22-31.

[32] Kim E, Xia Y, Whitesides GM. Micromolding in Capillaries: Applications in Materials Science. J. Am. Chem. Soc. 1996; 118:5722-5731. 


\section{Figures and tables Captions}

Table 1: Description of the investigated commercial basic silicate solutions. Suppliers: * Woellner ** Fluka Figure 1 : Typical SEM picture of a cross section of lithium silicate coating. The film thickness is about $150 \mathrm{~nm}$.

Figure 2 : a ${ }^{29} \mathrm{Si}$ NMR spectra of alkaline silicate solutions: (black) $\mathrm{K}(\mathrm{x}=0.33)$, (red) $\mathrm{Na}(\mathrm{x}=0.33)$ and (blue) $\mathrm{K} / \mathrm{Li}(\mathrm{x}=0.33)$.

b) FTIR spectra of (black) potassium silicate coatings ( $x=0.33$ ), (red) sodium silicate coatings $(x=0.33)$, (blue) potassium/lithium silicate coatings $(x=0.33)$ and (grey) TEOS-based coatings.

Straight line: at ambient temperature/Dashed line: after annealing at $450^{\circ} \mathrm{C} / 2 \mathrm{~h}$.

c) ${ }^{29} \mathrm{Si}$ NMR spectra of alkaline silicate solutions: (black) $\mathrm{Li}(\mathrm{x}=0.4)$ and (red) $\mathrm{Li}(\mathrm{x}=0.2)$.

d) FTIR spectra of (black) lithium silicate coatings $(x=0.4)$, (red) lithium silicate coatings $(x=0.2)$ and (grey) TEOS-based coatings.

Table 2 : Composition and repartition of $Q^{n}$ silicate species of dried/annealed silicate powders (obtained from ${ }^{29}$ Si solid-state NMR spectra).

Figure 3 : Evolution of the maximum value of the main SiOSi FTIR band while annealing the silicate coatings (for samples just after spin-coating and after two months of ageing) and comparison with the reference coating TEOS.

Figure 4 : Optical microscopy image of the samples surface two weeks $(a \rightarrow e)$ and three months $(f \rightarrow j)$ after deposition: $a / f)$ Sodium silicate, $x=0.33 \mathrm{~b} / \mathrm{g}$ ) Potassium silicate, $x=0.33 \mathrm{c} / \mathrm{h}$ ) Potassium/lithium silicate, $x=0.33 \mathrm{~d} / \mathrm{i})$ Lithium silicate, $x=0.2 \mathrm{e} / \mathrm{j})$ TEOS

Figure 5 : a) Grazing-incidence $\left(3^{\circ}\right)$ XRD diffractogramm of a 1 month aged sodium silicate coating; b) SEM image of a sodium silicate coating (1 month ageing) ([Si]=4.7M).

c) and d) FTIR spectra of a sodium silicate coating and TEOS-based coating after different times (1 week to 8 weeks) of ageing under ambient conditions. 
Figure 6 : a) FTIR spectra of a lithium silicate coating ( $x=0.2)$ just after coating (black) then water-washed (blue) and annealed at $450^{\circ} \mathrm{C} / 2 \mathrm{hrs}$ (dark blue) and comparison with a TEOS coating just after coating (grey) then annealed at $450^{\circ} \mathrm{C} / 2 \mathrm{hrs}$ (dark grey) b) FTIR spectra of various silicate coatings (water-washed then annealed at $450^{\circ} \mathrm{C}$ ) c) Refractive index at $600 \mathrm{~nm}$ (from ellipsometry measurements) for "fresh" silicate coatings, water-washed coatings then annealed at $450^{\circ} \mathrm{C}$. d) FTIR spectra of 6 months-old sodium silicate coatings at ambient temperature: (black) reference coating and (red) water-washed coating.

Figure 7 : a) $\mathrm{YVO}_{4}$ : Eu nanoparticles dispersed in water (left) and in a mix of water/lithium silicate after (right) after several days settling down b) Luminescent coatings of $\mathrm{YVO}_{4}$ :Eu nanoparticles dispersed in a mix of water/lithium silicate under UV excitation.

Figure 8 : SEM cross section picture of a mesoporous latex-templated lithium silicate film, and a top side picture of the film (inset).

Figure 9: SEM image of a structured Li0.2 coating obtained using the MIMIC process. Insert show the diffraction of white light from the periodic structure. 